\title{
Ricci curvature of integral submanifolds of an f.p.k.-space form
}

\author{
Mahmood Jaafari Matehkolaee
}

\begin{abstract}
In this paper, relationships between the Ricci curvature and the squared mean curvature for integral submanifolds of an f.p.k.-space form by a basic inequality, are studied. We show that if an integral submanifold of maximum dimension of an f.p.k.-space form satisfies the equality case, then it must be minimal.
\end{abstract}

\section{Introduction}

In words of B. Y. Chen, to "find simple relationships between the main extrinsic invariant and the main intrinsic invariants of a submanifold" is one of the basic problems in the theory of submanifolds ([7]). In this way, he established a relationship between sectional curvature function and the shape operator for submanifolds in real space forms [7] and another relationship between the Ricci curvature and the squared mean curvature [9]. Corresponding relationships have been proved for submanifolds of Sasakian space forms [18], C-totally real submanifolds in sasakian space forms [15], integral submanifolds of $S$-space forms [15], submanifolds of $S$-space forms [16].

On the other hand, for manifolds with an $f$-structure, D. E. Blair has introduced $S$-manifolds as the analogue of the Kähler structure in the almost complex case and of Sasakian structure in the almost contact case [3]. Also, f.p.k.space forms are introduced by M. Falcitelli and A. M. Pastore as generalization of Sasakian space forms [10].

Received by the editors in April 2013 - In revised form in August 2013.

Communicated by F. Bourgeois.

2010 Mathematics Subject Classification : 53C15, 53C25, 53D10.

Key words and phrases : f.p.k.-space form, Ricci curvature, $k$-Ricci curvature, Scalar curvature, Integral submanifold, $C$-totally real submanifold. 
The purpose of the present paper is to study Ricci tensor, sectional curvature and scalar curvature of submanifolds of a generalized f.p.k.-space form and obtain similar relationship to Chen's one mentioned above. In section 2, we state definitions of f.p.k.-space form and its curvature tensor. Section 3 is devoted to the study sectional curvature of submanifold of an f.p.k.-space form. In section 4, we investigate Ricci tensor and scalar curvature of submanifold of an f.p.k.-space form. In section 5, we study Chen's relationship between the Ricci curvature and the squared mean curvature for integral submanifolds. Finally, in section 6, we find minimality of integral submanifolds of maximum dimension.

\section{Preliminaries}

In the class of $f$-structures introduced in 1963 by Yano [26], particularly interesting are the so-called $f$-structures with complemented frames, also called globally framed $f$-structures or $f$-structures with parallelizable kernel (briefly f.p.k.-structures) ([3]). An f.p.k.-manifold is a manifold $M^{2 n+s}$ on which is defined an $f$-structure, that is a $(1,1)$-tensor field $\varphi$ satisfying $\varphi^{3}+\varphi=0$, of rank $2 n$, such that the subbundle $\operatorname{ker} \varphi$ is parallelizable. Then, there exists a global frame $\left\{\xi_{i}\right\}, i \in\{1, \ldots, s\}$, for the subbundle $\operatorname{ker} \varphi$, with dual 1-form $\eta^{i}$, satisfying $\varphi^{2}=-I+\eta^{i} \otimes \xi_{i}, \eta^{i}\left(\xi_{j}\right)=\delta_{j}^{i}$, from which $\varphi \xi_{i}=0, \eta^{i} \circ \varphi=0$ follow. An f.p.k.-structure on a manifold $M^{2 n+s}$ is said to be normal if the tensor field $N=$ $[\varphi, \varphi]+2 d \eta^{i} \otimes \xi_{i}$ vanishes, $[\varphi, \varphi]$ denoting the Nijenhuis torsion of $\varphi$. It is known that one can consider a Riemannian metric $g$ on $M^{2 n+s}$ associated with an f.p.k.structure $\left(\varphi, \xi_{i}, \eta^{i}\right)$, such that $g(\varphi X, \varphi Y)=g(X, Y)-\sum_{i=1}^{s} \eta^{i}(X) \eta^{i}(Y)$, for any $X, Y \in \Gamma\left(T^{2 n+s} M\right)$, and the structure $\left(\varphi, \xi_{i}, \eta^{i}, g\right)$ is then called a metric f.p.k.structure. Therefore, $T^{2 n+s} M$ splits as complementary orthogonal sum of its subbundles $\operatorname{Im} \varphi$ and $\operatorname{ker} \varphi$. We denote their respective differentiable distributions by $D$ and $D^{\perp}$.

Let $\Omega$ denote the 2-form on $M^{2 n+s}$ defined by $\Omega(X, Y)=g(X, \varphi Y)$, for any $X, Y \in \Gamma\left(T^{2 n+s} M\right)$.

Several subclasses have been studied from different points of view $([2,3])$, also dropping the normality condition and, in this case, the term almost precedes the name of the considered structures or manifolds. As in ([3]), a metric f.p.k.structure is said a $K$-structure if it is normal and the fundamental 2-form $\Omega$ is closed; a manifold with a $K$-structure is called a $K$-manifold. In particular, if $d \eta^{i}=\Omega$, for all $i \in\{1, \ldots, s\}$, the $K$-structure is said an $S$-structure and $M^{2 n+s}$ an $S$-manifold. Finally, if $d \eta^{i}=0$ for all $i \in\{1, \ldots, s\}$, then the $K$-structure is called a $C$-structure and $M^{2 n+s}$ is said a $C$-manifold. Obviously, if $s=1$, a $K$-manifold $M^{2 n+1}$ is a quasi Sasakian manifold, a $C$-manifold is a cosymplectic manifold and an $S$-manifold is a Sasakian manifold.

We recall that the Levi-Civita connection $\nabla$ of a metric $f . p . k$--manifold satisfies 
the following formula ([3]):

$$
\begin{aligned}
2 g\left(\left(\nabla_{X} \varphi\right) Y, Z\right)= & 3 d \Omega(X, \varphi Y, \varphi Z)-3 d \Omega(X, Y, Z) \\
+ & g(N(Y, Z), \varphi X)+N_{j}^{(2)}(Y, Z) \eta^{j} X \\
+ & d \eta^{j}(\varphi Y, X) \eta^{j}(Z)-2 d \eta^{j}(\varphi Z, X) \eta^{j}(Y),
\end{aligned}
$$

where $N_{j}^{(2)}$ is given by $N_{j}^{(2)}(X, Y)=2 d \eta^{j}(\varphi X, Y)-2 d \eta^{j}(\varphi Y, X)$.

Furthermore, for $S$-manifolds we have $\nabla_{X} \xi_{j}=-\varphi X, j=1, \ldots, s,([4])$. Putting $\bar{\xi}=\sum_{j=1}^{s} \xi_{j}, \bar{\eta}=\sum_{j=1}^{s} \eta_{j}$ is its dual form with respect to $g$ and:

$$
\left(\nabla_{X} \varphi\right) Y=g(\varphi X, \varphi Y) \bar{\xi}+\bar{\eta}(Y) \varphi^{2} X
$$

We remark that (2.2) together with $£_{\xi_{i}} g=0$ and $£_{\xi_{i}} \eta^{j}=0, i, j \in\{1, \ldots, s\}$, characterizes the $S$-manifolds among the metric f.p.k.-manifolds.

A metric f.p.k.-manifold $\left(M^{2 n+s}, \varphi, \xi_{i}, \eta^{i}, g\right)$ has pointwise constant (p.c.) $\varphi$-sectional curvature if at any $p \in M^{2 n+s}, c(p)=R_{p}(X, \varphi X, X, \varphi X)$ does not depend on the $\varphi$-section spanned by $\{X, \varphi X\}$, for any unit $X \in D_{p}$. Several results involving the pointwise constancy of the $\varphi$-sectional curvatures of an almost contact metric manifold (i.e. for $s=1$ ) are recently obtained in [1, 14]. We refer to [5] for a systematic exposition of the classical curvature results on contact metric manifolds.

We recall some known results.

Proposition 2.1. ([18, 19]) A Sasaki manifold $\left(M^{2 n+1}, \varphi, \xi, \eta, g\right)$ has p.c. $\varphi$-sectional curvature $c$ if and only if its curvature tensor field verifies:

$$
\begin{aligned}
R(X, Y, Z) & =\frac{1}{4}(c+3)\{g(Y, Z) X-g(X, Z) Y\} \\
& +\frac{1}{4}(c-1)\{g(X, \varphi Z) \varphi Y-g(Y, \varphi Z) \varphi X+2 g(X, \varphi Y) \varphi Z \\
& +\eta(X) \eta(Z) Y-\eta(Y) \eta(Z) X+g(X, Z) \eta(Y) \xi-g(Y, Z) \eta(X) \xi\}
\end{aligned}
$$

for any $X, Y, Z$ tangent to $M^{2 n+1}$.

A Sasaki manifold $M^{2 n+1}$ with constant $\varphi$-sectional curvature $c \in \mathbb{R}$ is called a Sasakian space form and denoted by $M^{2 n+1}(c)$. It is well known that, if $n \geq 2$, a Sasaki manifold $M^{2 n+1}$ with p.c. $\varphi$-sectional curvature $c$ is a Sasakian space form. As examples of Sasakian space forms we mention $\mathbb{R}^{2 n+1}$ and $S^{2 n+1}$, with standard Sasakian structures ([2]).

Definition 2.2. ([3]) An almost contact metric manifold $\left(M^{2 n+1}, \varphi, \xi, \eta, g\right)$ is a generalized Sasakian space form, denoted by $\left(M^{2 n+1}, f_{1}, f_{2}, f_{3}\right)$, if it admits three smooth functions $f_{1}, f_{2}, f_{3}$ such that its curvature tensor field verifies, for any $X, Y, Z \in T M$ :

$$
\begin{aligned}
R(X, Y, Z) & =f_{1}\{g(Y, Z) X-g(X, Z) Y\} \\
& +f_{2}\{g(X, \varphi Z) \varphi Y-g(Y, \varphi Z) \varphi X+2 g(X, \varphi Y) \varphi Z\} \\
& +f_{3}\{\eta(X) \eta(Z) Y-\eta(Y) \eta(Z) X+g(X, Z) \eta(Y) \xi-g(Y, Z) \eta(X) \xi\},
\end{aligned}
$$

Remark 2.3. Any generalized Sasakian space form has p.c. $\varphi$-sectional curvature $c=f_{1}+3 f_{2}$. Obviously, a Sasaki manifold of p.c. $\varphi$-sectional curvature $c$ satisfies (2.4) with $f_{1}=\frac{1}{4}(c+3)$, and $f_{2}=f_{3}=\frac{1}{4}(c-1)$. A cosymplectic manifold with p.c. $\varphi$-sectional curvature c satisfies (2.4) with $f_{1}=f_{2}=f_{3}=\frac{1}{4}$ c. 
Proposition 2.4. ([3]) An S-manifold $M^{2 n+s}$ has p.c. $\varphi$-sectional curvature $c$ if and only if its curvature tensor field verifies:

$$
\begin{aligned}
R(X, Y, Z) & =\frac{1}{4}(c+3 s)\left\{g(\varphi X, \varphi Z) \varphi^{2} Y-g(\varphi Y, \varphi Z) \varphi^{2} X\right\} \\
& +\frac{1}{4}(c-s)\{g(Z, \varphi Y) \varphi X-g(Z, \varphi X) \varphi Y+2 g(X, \varphi Y) \varphi Z\} \\
& +\left\{\bar{\eta}(X) \bar{\eta}(Z) \varphi^{2} Y-\bar{\eta}(Y) \bar{\eta}(Z) \varphi^{2} X+g(\varphi Y, \varphi Z) \bar{\eta}(Y) \bar{\xi}\right. \\
& -g(\varphi X, \varphi Z) \bar{\eta}(X) \bar{\xi}\} .
\end{aligned}
$$

for any $X, Y, Z$ tangent to $M^{2 n+1}$.

An $S$-manifold $M^{2 n+s}$ with constant $\varphi$-sectional curvature $c \in \mathbb{R}$ is called an $S$-space form and denoted by $M^{2 n+s}(c)$. Moreover, it is also well known that if $n \geq 2$ then an $S$-manifold with p.c. $\varphi$-sectional curvature $c$ is an $S$-space form. We remark that for $s=1$ (2.5) reduces to (2.3).

Definition 2.5. In [19], Oubiña introduced the notion of a trans-Sasakian manifold. An almost contact metric manifold $M$ is called trans-Sasakian manifold if there exist two functions $\alpha$ and $\beta$ on $M$ such that ([22]):

$$
\left(\nabla_{X} \varphi\right)(Y)=\alpha\{g(X, Y) \xi-\eta(Y) X\}+\beta\{g(\varphi X, Y) \xi-\eta(Y) \varphi X\}
$$

for vector fields $X, Y$ on $M$. From (2.6) it is easy to see that:

$$
\nabla_{X} \xi=-\alpha \varphi X+\beta(X-\eta(X)) \xi .
$$

In particular, if $\beta=0$, then $M$ is said to be an $\alpha$-Sasakian manifold. Sasakian manifolds appear as examples of $\alpha$-Sasakian manifolds with $\alpha=1$.

On the other hand, if $\alpha=0$, then $M$ is said to be a $\beta$-Kenmotsu manifold. Kenmotsu manifolds, defined in [14], are particular examples with $\beta=1$.

Another important kind of trans-Sasakian manifolds is that of cosymplectic manifolds obtained for $\alpha=\beta=0$.

Proposition 2.6. ([13]) An almost contact metric manifold is said to be an almost $C(\alpha)$-manifold if its Riemannian curvature tensor verifies:

$$
\begin{aligned}
R(X, Y, Z, W) & =R(X, Y, \varphi Z, \varphi W)+\alpha\{g(X, W) g(Y, Z)-g(X, Z) g(Y, W) \\
& +g(X, \varphi Z) g(Y, \varphi W)-g(X, \varphi W) g(Y, \varphi Z)\}
\end{aligned}
$$

for vector fields $X, Y, Z, W$ on $M$, where $\alpha$ is a real number. Moreover, if such a manifold has constant $\varphi$-sectional curvature equal to $c$, then its curvature tensor is given by:

$$
\begin{aligned}
R(X, Y) Z & =\frac{1}{4}\left(c+3 \alpha^{2}\right)\{g(Y, Z) X-g(X, Z) Y\} \\
& +\frac{1}{4}\left(c-\alpha^{2}\right)\{g(X, \varphi Z) \varphi Y-g(Y, \varphi Z) \varphi X+2 g(X, \varphi Y) \varphi Z \\
& +\eta(X) \eta(Z) Y-\eta(Y) \eta(Z) X+g(X, Z) \eta(Y) \xi-g(Y, Z) \eta(X) \xi\}
\end{aligned}
$$

and so, it is a generalized Sasakian space form with $f_{1}=\frac{1}{4}\left(c+3 \alpha^{2}\right)$ and $f_{2}=f_{3}=$ $\frac{1}{4}\left(c-\alpha^{2}\right)$.

Example A. The products between a complex space form and a one dimensional manifold are the main examples of cosymplectic space forms, which are 
often seen as the odd dimensional version of complex space forms. We mention $\mathbb{C} P^{n} \times \mathbb{R}$ and $\mathbb{C} H^{n} \times \mathbb{R}$ as special examples.

(See [14) A Kenmotsu manifold is a warped product $I \times_{f} N$ of an interval $I$ and a Kähler manifold $N$ with warping function $f(t)=s e^{t}$, where $s$ is a nonzero constant and a Kenmotsu space form is a space of constant curvature -1, and then it is locally hyperbolic space.

There exists no connected Kenmotsu space form of dimension $\geq 5$. Examples of Kenmotsu space forms are not known so far and according to D. E. Blair (in a private conversation), one doubts that there are any.

For example of almost $C(\alpha)$-manifold we observe that Sasakian manifolds are C(1)-manifolds.

Pseudo-umbilical, totally contact umbilical, totally contact geodesic, totally umbilical and totally geodesic hypersurfaces of an $S$-space form are also $S$-space forms.

Let $\mathcal{F}$ denote any set of smooth function $F_{i j}$ on $M^{2 n+s}$ such that $F_{i j}=F_{j i}$ for any $i, j \in\{1,2, \ldots, s\}$.

Definition 2.7. ([10]) $A$ (generalized) f.p.k.-space form denoted by $M^{2 n+s}\left(F_{1}, F_{2}, \mathcal{F}\right)$, is a metric $f$.p.k.-manifold $\left(M^{2 n+s}, \varphi, \xi_{i}, \eta^{i}, g\right)$ which admits smooth functions $F_{1}, F_{2}, \mathcal{F}$ such that its curvature tensor field verifies:

$$
\begin{aligned}
R(X, Y, Z) & =F_{1}\left\{g(\varphi X, \varphi Z) \varphi^{2} Y-g(\varphi Y, \varphi Z) \varphi^{2} X\right\} \\
& +F_{2}\{g(Z, \varphi Y) \varphi X-g(Z, \varphi X) \varphi Y+2 g(X, \varphi Y) \varphi Z\} \\
& +\sum_{i, j=1}^{s} F_{i j}\left\{\eta^{i}(X) \eta^{j}(Z) \varphi^{2} Y-\eta^{i}(Y) \eta^{j}(Z) \varphi^{2} X+\eta^{i}(X) \xi_{j} g(\varphi Y, \varphi Z)\right. \\
& \left.-\eta^{i}(Y) \xi_{j} g(\varphi X, \varphi Z)\right\} .
\end{aligned}
$$

For $s=1$, we obtain a generalized Sasakian space form $M^{2 n+1}\left(f_{1}, f_{2}, f_{3}\right)$ with $f_{1}=F_{1}, f_{2}=F_{2}$ and $f_{3}=F_{1}-F_{11}$. In particular, if the given structure is either Sasakian, or Kenmotsu, or possibly cosymplectic, then (2.8) holds with $F_{11}=1$, $F_{1}=\frac{1}{4}(c+3), F_{2}=\frac{1}{4}(c-1)$ and $f_{3}=F_{1}-F_{11}=\frac{1}{4}(c-1)=f_{2}$ in the first case, $F_{11}=-1, F_{1}=\frac{1}{4}(c-3), F_{2}=\frac{1}{4}(c+1)$ and $f_{3}=F_{1}-F_{11}=\frac{1}{4}(c+1)=f_{2}$ in the second case, and $F_{11}=0, F_{1}=\frac{1}{4} c, F_{2}=\frac{1}{4} c$ and $f_{3}=\frac{1}{4} c$ in the last case.

Definition 2.8. ([20]) An almost Hermitian manifold $M^{2 n}$ is called a generalized complex space form $M\left(f_{1}, f_{2}\right)$ if its Riemannian curvature tensor $R$ satisfies:

$$
\begin{aligned}
R(X, Y, Z)=f_{1}\{g(Y, Z) X-g(X, Z) Y\}+ \\
\qquad f_{2}\{g(X, J Z) J Y-g(Y, J Z) J X+2 g(X, J Y) J Z\},
\end{aligned}
$$

for all $X, Y, Z \in T M$, where $f_{1}$ and $f_{2}$ are smooth functions on $M$.

Example B. Any Kähler manifold with constant holomorphic sectional curvature is a generalized complex space form. This is also true for any almost Hermitian manifold with constant sectional curvature, as for example 6-dimensional sphere which is not Kähler manifold. In fact, These cases are the only possible ones when $2 n \geq 6$. 
Example C. (see [10]) Considers a 4-dimensional generalized complex spaceform $(\bar{M}, J, \bar{g})$ with $\bar{R}=f_{1} \pi_{1}+f_{2} \pi_{2}, f_{2}$ nowhere vanishing and non constant, and the Riemannian product $M^{4+s}=\bar{M} \times \mathbb{R}^{s}$, with metric $g=\bar{g}+g_{0}, g_{0}$ being the standard metric on $R^{s}$. Put $\xi_{k}=\frac{\partial}{\partial x^{k}}$ and $\varphi \bar{X}=J \bar{X}$ for $\bar{X} \in T \bar{M}, \varphi \xi_{k}=0$ for any $k \in\{1, \ldots, s\}$ and $\eta^{k} X=g\left(X, \xi_{k}\right)$. Then, $\left(M^{4+s}, \varphi, \xi_{k}, \eta^{k}, g\right)$ is a generalized f.p.k.-space form.

Let $M$ be an $m$-dimensional submanifold immersed in a generalized f.p.k.space form $\bar{M}^{2 n+s}$. The Gauss-Weingarten formulas are given by:

$$
\begin{gathered}
\bar{\nabla}_{X} Y=\nabla_{X} Y+h(X, Y) ; \quad X, Y \in T M, \\
\bar{\nabla}_{X} N=-A_{N} X+\nabla_{X}^{\perp} N ; \quad X \in T M, N \in T^{\perp} M,
\end{gathered}
$$

where $\nabla^{\perp}$ is the connection in the normal bundle, $h$ is the second fundamental form of $M$ and $A_{N}$ the Weingarten endomorphism associated with $N$. Then $A_{N}$ and $h$ are related by:

$$
g\left(A_{N} X, Y\right)=g(h(X, Y), N)
$$

We denote by $\bar{R}$ and $R$ the curvature tensor fields associated with $\bar{\nabla}$ and $\nabla$, respectively. The Gauss equation is given by:

$$
\bar{R}(X, Y, Z, W)=R(X, Y, Z, W)+g(h(X, Z), h(Y, W))-g(h(X, W), h(Y, Z)),
$$

where $X, Y, Z, W$ belong to TM.

\section{Sectional Curvature of Submanifolds}

Let $M$ be a submanifold of a generalized f.p.k.-space form $\bar{M}^{2 n+s}\left(F_{1}, F_{2}, \mathcal{F}\right)$. Then from equation of Gauss we have:

$$
\begin{aligned}
R(X, Y, Z, W)= & \bar{R}(X, Y, Z, W)-g(h(X, Z), h(Y, W))+g(h(X, W), h(Y, Z)) \\
= & F_{1}\left\{g(\varphi X, \varphi Z) g\left(\varphi^{2} Y, W\right)-g(\varphi Y, \varphi Z) g\left(\varphi^{2} X, W\right)\right\} \\
+ & F_{2}\{g(Z, \varphi Y) g(\varphi X, W)-g(Z, \varphi X) g(\varphi Y, W)+ \\
& 2 g(X, \varphi Y) g(\varphi Z, W)\} \\
+ & \sum_{i, j=1}^{S} F_{i j}\left\{\eta^{i}(X) \eta^{j}(Z) g\left(\varphi^{2} Y, W\right)-\eta^{i}(Y) \eta^{j}(Z) g\left(\varphi^{2} X, W\right)+\right. \\
& \left.\eta^{i}(X) \eta^{j}(W) g(\varphi Y, \varphi Z)-\eta^{i}(Y) \eta^{j}(W) g(\varphi X, \varphi Z)\right\} \\
& -g(h(X, Z), h(Y, W))+g(h(X, W), h(Y, Z))
\end{aligned}
$$

for any $X, Y, Z, W$ tangent to $M$.

Let $K_{M}(X, Y)$ be the sectional curvature determined by orthonormal vectors 
$X$ and $Y$. Then from equation (3.1) we have:

$$
\begin{aligned}
K_{M}(X, Y) & =g(h(X, X), h(Y, Y))-\|h(X, Y)\|^{2}+3 F_{2} g^{2}(X, \varphi Y) \\
& +F_{1}\left\{\left(1-\sum_{k=1}^{s} \eta^{k}(X)^{2}\right)\left(1-\sum_{k=1}^{s} \eta^{k}(Y)^{2}\right)-\left(\sum_{k=1}^{s} \eta^{k}(X) \eta^{k}(Y)\right)^{2}\right\} \\
& +\sum_{i, j=1}^{s} F_{i j}\left\{\eta^{i}(X) \eta^{j}(X)\left(1-\sum_{k=1}^{s} \eta^{k}(Y)^{2}\right)+\eta^{i}(Y) \eta^{j}(Y)\left(1-\sum_{k=1}^{s} \eta^{k}(X)^{2}\right)\right. \\
& \left.+2 \eta^{i}(X) \eta^{j}(Y) \sum_{k=1}^{s} \eta^{k}(X) \eta^{k}(Y)\right\} .
\end{aligned}
$$

Thus we have:

Theorem 3.1. Let $M$ be a submanifold of a generalized f.p.k.-space form $\bar{M}^{2 n+s}\left(F_{1}, F_{2}, \mathcal{F}\right)$. Then the sectional curvature of $M$ determined by orthogonal tangent vectors $\{X, Y\}$ is given by:

$$
\begin{aligned}
K_{M}(X, Y) & =g(h(X, X), h(Y, Y))-\|h(X, Y)\|^{2}+3 F_{2} g^{2}(X, \varphi Y) \\
& +F_{1}\left\{\left(1-\sum_{k=1}^{s} \eta^{k}(X)^{2}\right)\left(1-\sum_{k=1}^{s} \eta^{k}(Y)^{2}\right)-\left(\sum_{k=1}^{s} \eta^{k}(X) \eta^{k}(Y)\right)^{2}\right\} \\
& +\sum_{i, j=1}^{s} F_{i j}\left\{\eta^{i}(X) \eta^{j}(X)\left(1-\sum_{k=1}^{s} \eta^{k}(Y)^{2}\right)+\eta^{i}(Y) \eta^{j}(Y)\left(1-\sum_{k=1}^{s} \eta^{k}(X)^{2}\right)\right. \\
& \left.+2 \eta^{i}(X) \eta^{j}(Y) \sum_{k=1}^{s} \eta^{k}(X) \eta^{k}(Y)\right\} .
\end{aligned}
$$

From this we have the following corollaries for the sectional curvature of submanifold determined by orthonormal tangent vectors $\{X, Y\}$.

Corollary 3.2. The sectional curvature of a submanifold of an S-space form $\bar{M}^{2 n+s}(c)$ is given by:

$$
\begin{aligned}
K_{M}(X, Y) & =g(h(X, X), h(Y, Y))-\|h(X, Y)\|^{2}+\frac{3}{4}(c-s) g^{2}(X, \varphi Y) \\
& +\frac{1}{4}(c+3 s)\left\{\left(1-\sum_{k=1}^{s} \eta^{k}(X)^{2}\right)\left(1-\sum_{k=1}^{s} \eta^{k}(Y)^{2}\right)-\left(\sum_{k=1}^{s} \eta^{k}(X) \eta^{k}(Y)\right)^{2}\right\} \\
& +\sum_{i, j=1}^{s}\left\{\eta^{i}(X) \eta^{j}(X)\left(1-\sum_{k=1}^{s} \eta^{k}(Y)^{2}\right)+\eta^{i}(Y) \eta^{j}(Y)\left(1-\sum_{k=1}^{s} \eta^{k}(X)^{2}\right)\right. \\
& \left.+2 \eta^{i}(X) \eta^{j}(Y) \sum_{k=1}^{s} \eta^{k}(X) \eta^{k}(Y)\right\} .
\end{aligned}
$$

Proof. We will get the result by using $F_{i j}=1 ; \forall 1 \leq i, j \leq s, F_{1}=\frac{1}{4}(c+3 s)$, $F_{2}=\frac{1}{4}(c-s)$ in (3.2).

Corollary 3.3. The sectional curvature of a submanifold of a generalized Sasakian space form $\bar{M}(c)$ is given by:

$$
\begin{array}{r}
K_{M}(X, Y)=g(h(X, X), h(Y, Y))-\|h(X, Y)\|^{2}+f_{1}+3 f_{2} g^{2}(X, \varphi Y)- \\
f_{3}\left(\eta^{2}(X)+\eta^{2}(Y)\right) .
\end{array}
$$


Proof. We will get the result by using $s=1, F_{1}=f_{1}, F_{2}=f_{2}, F_{11}=f_{1}-f_{3}$ in (3.2).

Corollary 3.4. The sectional curvature of a submanifold of a Sasakian space form $\bar{M}(c)$ is given by:

$$
\begin{aligned}
K_{M}(X, Y) & =g(h(X, X), h(Y, Y))-\|h(X, Y)\|^{2}+\frac{1}{4}(c+3) \\
& +\frac{1}{4}(c-1)\left(3 g^{2}(X, \varphi Y)-\eta^{2}(X)-\eta^{2}(Y)\right) .
\end{aligned}
$$

Proof. We get the result by using $f_{1}=\frac{1}{4}(c+3), f_{2}=f_{3}=\frac{1}{4}(c-1)$ in (3.4).

Corollary 3.5. The sectional curvature of a submanifold of a Kenmotsu space form $\bar{M}(c)$ is given by:

$$
\begin{aligned}
K_{M}(X, Y) & =g(h(X, X), h(Y, Y))-\|h(X, Y)\|^{2}+\frac{1}{4}(c-3) \\
& +\frac{1}{4}(c+1)\left(3 g^{2}(X, \varphi Y)-\eta^{2}(X)-\eta^{2}(Y)\right) .
\end{aligned}
$$

Proof. We get the result by using $f_{1}=\frac{1}{4}(c-3), f_{2}=f_{3}=\frac{1}{4}(c+1)$ in (3.4).

Corollary 3.6. The sectional curvature of a submanifold of a cosymplectic space form $\bar{M}(c)$ is given by:

$$
\begin{aligned}
K_{M}(X, Y) & =g(h(X, X), h(Y, Y))-\|h(X, Y)\|^{2} \\
& +\frac{1}{4} c\left(1+3 g^{2}(X, \varphi Y)-\eta^{2}(X)-\eta^{2}(Y)\right)
\end{aligned}
$$

Proof. By taking $f_{1}=f_{2}=f_{3}=\frac{1}{4} c$ in (3.4), we obtain the above.

Corollary 3.7. The sectional curvature of a submanifold of an almost $C(\alpha)$-manifold $\bar{M}(c)$ is given by:

$$
\begin{aligned}
K_{M}(X, Y) & =g(h(X, X), h(Y, Y))-\|h(X, Y)\|^{2}+\frac{1}{4}\left(c+3 \alpha^{2}\right) \\
& +\frac{1}{4}\left(c-\alpha^{2}\right)\left(3 g^{2}(X, \varphi Y)-\eta^{2}(X)-\eta^{2}(Y)\right)
\end{aligned}
$$

Proof. We getting $f_{1}=\frac{1}{4}\left(c+3 \alpha^{2}\right), f_{2}=f_{3}=\frac{1}{4}\left(c-\alpha^{2}\right)$ in (3.4), we obtain (3.8). 


\section{The Ricci Tensors and Scalar Curvature of a Submanifold}

Let $M$ be a submanifold of a generalized f.p.k.-space form $\bar{M}^{2 n+s}\left(F_{1}, F_{2}, \mathcal{F}\right)$. Then it is straightforward to calculate the Ricci tensor of $M$ as following:

$$
\begin{aligned}
& \operatorname{Ric}(X, Y)=\sum_{k=1}^{n} g\left(R\left(e_{k}, X\right) Y, e_{k}\right) \\
& =\sum_{k=1}^{n} F_{1}\left\{g\left(\varphi e_{k}, \varphi Y\right) g\left(\varphi^{2} X, e_{k}\right)-g(\varphi X, \varphi Y) g\left(\varphi^{2} e_{k}, e_{k}\right)\right\} \\
& +\sum_{k=1}^{n} F_{2}\left\{g(Y, \varphi X) g\left(\varphi e_{k}, e_{k}\right)-g\left(Y, \varphi e_{k}\right) g\left(\varphi X, e_{k}\right)\right. \\
& \left.+2 g\left(e_{k}, \varphi X\right) g\left(\varphi Y, e_{k}\right)\right\} \\
& +\sum_{k=1}^{n} \sum_{i, j=1}^{s} F_{i j}\left\{\eta^{i}\left(e_{k}\right) \eta^{j}(Y) g\left(\varphi^{2} X, e_{k}\right)-\eta^{i}(X) \eta^{j}(Y) g\left(\varphi^{2} X, e_{k}\right)\right. \\
& \left.+\eta^{i}\left(e_{k}\right) \eta^{j}\left(e_{k}\right) g(\varphi X, \varphi Y)-\eta^{i}(X) \eta^{j}\left(e_{k}\right) g\left(\varphi e_{k}, \varphi Y\right)\right\} \\
& \text { - } g\left(h\left(e_{k}, Y\right), h\left(X, e_{k}\right)\right)+g\left(h\left(e_{k}, e_{k}\right), h(X, Y)\right) \\
& =F_{1}\left\{-g(\varphi X, \varphi Y)-g(\varphi X, \varphi Y) \sum_{k=1}^{n}\left(-1+\sum_{\alpha=1}^{s}\left(\eta^{\alpha}\left(e_{k}\right)\right)^{2}\right)\right\} \\
& +3 F_{2}\left\{g(X, Y)-\sum_{\alpha=1}^{s} \eta^{\alpha}(X) \eta^{\alpha}(Y)\right\} \\
& +\sum_{i, j=1}^{s} F_{i j}\left\{-\sum_{k=1}^{n} \eta^{i}\left(e_{k}\right) \eta^{j}(Y) g\left(\varphi X, \varphi e_{k}\right)+\eta^{i}(X) \eta^{j}(Y) \sum_{k=1}^{n} g\left(\varphi e_{k}, \varphi e_{k}\right)\right. \\
& \left.+g(\varphi X, \varphi Y) \sum_{k=1}^{n} \eta^{i}\left(e_{k}\right) \eta^{j}\left(e_{k}\right)-\eta^{i}(X) \sum_{k=1}^{n} \eta^{j}\left(e_{k}\right) g\left(\varphi Y, \varphi e_{k}\right)\right\} \\
& +\sum_{k=1}^{n}\left[g\left(h\left(e_{k}, e_{k}\right), h(X, X)\right)-g\left(h\left(X, e_{k}\right), h\left(Y, e_{k}\right)\right)\right] \\
& =F_{1}(n-s-1)\left(g(X, Y)-\sum_{\alpha=1}^{s} \eta^{\alpha}(X) \eta^{\alpha}(Y)\right) \\
& +3 F_{2}\left(g(X, Y)-\sum_{\alpha=1}^{s} \eta^{\alpha}(X) \eta^{\alpha}(Y)\right) \\
& +\sum_{i, j=1}^{s} F_{i j}(n-s) \eta^{i}(X) \eta^{j}(Y)+\left(\sum_{i=1}^{s} F_{i i}\right)\left(g(X, Y)-\sum_{\alpha=1}^{s} \eta^{\alpha}(X) \eta^{\alpha}(Y)\right) \\
& +\sum_{k=1}^{n}\left[g\left(h(X, X), h\left(e_{k}, e_{k}\right)\right)-g\left(h\left(X, e_{k}\right), h\left(Y, e_{k}\right)\right)\right] \text {. }
\end{aligned}
$$

Also, the scalar curvature $\rho$ of a submanifold $M$ of $\bar{M}^{2 n+s}\left(F_{1}, F_{2}, \mathcal{F}\right)$ is then 
given by:

$$
\begin{gathered}
\rho=\sum_{t=1}^{n} \operatorname{Ric}\left(e_{t}, e_{t}\right)=\left(F_{1}(n-s-1)+3 F_{2}\right)\left(\sum_{t=1}^{n} g\left(e_{t}, e_{t}\right)-\sum_{t=1}^{n} \sum_{\alpha=1}^{s}\left(\eta^{\alpha}\left(e_{t}\right)\right)^{2}\right) \\
+\sum_{i, j=1}^{s} F_{i j}(n-s) \sum_{t=1}^{n} \eta^{i}\left(e_{t}\right) \eta^{j}\left(e_{t}\right)+\left(\sum_{i=1}^{s} F_{i i}\right)\left(\sum_{t=1}^{n} g\left(e_{t}, e_{t}\right)-\sum_{t=1}^{n} \sum_{\alpha=1}^{s}\left(\eta^{\alpha}\left(e_{t}\right)\right)^{2}\right) \\
+\sum_{t=1}^{n} \sum_{k=1}^{n}\left[g\left(h\left(e_{t}, e_{t}\right), h\left(e_{k}, e_{k}\right)\right)-g\left(h\left(e_{t}, e_{k}\right), h\left(e_{t}, e_{k}\right)\right)\right] \\
=(n-s)\left((n-s-1) F_{1}+3 F_{2}\right)+(n-s) \sum_{i=1}^{s} F_{i i}+(n-s) \sum_{i=1}^{s} F_{i i} \\
+\sum_{i, j=1}^{n}\left[g\left(h\left(e_{i}, e_{i}\right), h\left(e_{j}, e_{j}\right)\right)-g\left(h\left(e_{i}, e_{j}\right), h\left(e_{i}, e_{j}\right)\right)\right]
\end{gathered}
$$

Thus, we obtain following:

Theorem 4.1. Let $M$ be a submanifold of a generalized f.p.k.-space form $\bar{M}^{2 n+s}\left(F_{1}, F_{2}, \mathcal{F}\right)$. Then the Ricci tensor and scalar curvature of $M$ (resp.) are given by:

$$
\begin{aligned}
\operatorname{Ric}(X, Y) & =\left((n-s-1) F_{1}+3 F_{2}+\sum_{i=1}^{s} F_{i i}\right) g(\varphi X, \varphi Y) \\
& +(n-s) \sum_{i, j=1}^{s} F_{i j} \eta^{i}(X) \eta^{j}(Y) \\
& +\sum_{k=1}^{n}\left[g\left(h(X, X), h\left(e_{k}, e_{k}\right)\right)-g\left(h\left(X, e_{k}\right), h\left(Y, e_{k}\right)\right)\right]
\end{aligned}
$$

and:

$$
\begin{aligned}
\rho=(n-s)\left((n-s-1) F_{1}\right. & \left.+3 F_{2}+2 \sum_{i=1}^{s} F_{i i}\right) \\
& +\sum_{i, j=1}^{n}\left[g\left(h\left(e_{i}, e_{i}\right), h\left(e_{j}, e_{j}\right)\right)-g\left(h\left(e_{i}, e_{j}\right), h\left(e_{i}, e_{j}\right)\right)\right]
\end{aligned}
$$

\section{Ricci curvature of integral submanifolds}

Let $M$ be an $n$-dimensional Riemannian manifold. Let $\left\{e_{1}, \ldots, e_{k}\right\}, 2 \leq k \leq n$, be an orthonormal basis of a $k$-plane section $\Pi_{k}$ of $T_{p}(M)$. If $k=n$ then $\Pi_{n}=T_{p}(M)$, and if $k=2$ then $\Pi_{2}$ is a plane section of $T_{p}(M)$. For a fixed $i \in\{1, \ldots, k\}$, a $k$ Ricci curvature of $\Pi_{k}$ at $e_{i}$, denoted $\operatorname{Ric}_{\Pi_{k}}\left(e_{i}\right)$, is defined by [9]:

$$
\operatorname{Ric}_{\Pi_{k}}\left(e_{i}\right)=\sum_{j \neq i}^{k} K_{i j},
$$

where $K_{i j}$ is the sectional curvature of the plane section spanned by $e_{i}$ and $e_{j}$. An $n$-Ricci curvature $\operatorname{Ric}_{T_{p}(M)}\left(e_{i}\right)$ is the usual Ricci curvature of $e_{i}$, denoted $\operatorname{Ric}\left(e_{i}\right)$. Thus for any orthonormal basis $\left\{e_{1}, \ldots, e_{n}\right\}$, we have:

$$
\operatorname{Ric}_{T_{p}(M)}\left(e_{i}\right)=\operatorname{Ric}\left(e_{i}\right)=\sum_{j \neq i}^{n} K_{i j} \text {. }
$$


The scalar curvature $\rho\left(\Pi_{k}\right)$ of the $k$-plane section $\Pi_{k}$ is given by:

$$
\rho\left(\Pi_{k}\right)=\sum_{1 \leq i<j \leq k} K_{i j} .
$$

Geometrically, $\rho\left(\Pi_{k}\right)$ is the scalar curvature of the image $\exp _{p}\left(\Pi_{k}\right)$ of $\Pi_{k}$ at $p$ under the exponential map at $p$. The scalar curvature $\rho(p)$ of $M$ at $p$ is identical with the scalar curvature of the tangent space $T_{p} M$ of $M$ at $p$, that is, $\rho(p)=$ $\rho\left(T_{p} M\right)$.

Let $M$ be an $n$-dimensional submanifold of an $m$-dimensional Riemannian manifold $\bar{M}$ equipped with a Riemannian metric $\bar{g}$. The relative null space of $M$ at $p$ is defined by [9]:

$$
\mathcal{N}_{p}=\left\{X \in T_{p} M: h(X, Y)=0, \forall Y \in T_{p} M\right\},
$$

which is also known as the kernel of the second fundamental form at $p$ [8].

Now, let $\left\{e_{1}, \ldots, e_{n}\right\}$ be an orthonormal basis of the tangent space $T_{p} M$ and $e_{r}$ belongs to an orthonormal basis $\left\{e_{n+1}, \ldots, e_{m}\right\}$ of the normal space $T_{p}^{\perp} M$. We put:

$$
h_{i j}^{r}=g\left(h\left(e_{i}, e_{j}\right), e_{r}\right) \text {, }
$$

and:

$$
\|h\|^{2}=g\left(h\left(e_{i}, e_{j}\right), h\left(e_{i}, e_{j}\right)\right) .
$$

Let $K_{i j}$ and $\bar{K}_{i j}$ denote the sectional curvature of the plane section spanned by $e_{i}$ and $e_{j}$ at $p$ in the submanifold $M$ and in the ambient manifold $\bar{M}$, respectively. Thus, we can say that $K_{i j}$ and $\bar{K}_{i j}$ are the "intrinsic" and "extrinsic" sectional curvature of the $\operatorname{Span}\left\{e_{i}, e_{j}\right\}$ at $p$. In view of Gauss equation (2.9), we get:

$$
K_{i j}=\bar{K}_{i j}+\sum_{r=n+1}^{m}\left(h_{i i}^{r} h_{j j}^{r}-\left(h_{i j}^{r}\right)^{2}\right) .
$$

From (5.3) it follows that:

$$
2 \rho(p)=2 \bar{\rho}\left(T_{p} M\right)+n^{2}\|\mu\|^{2}-\|h\|^{2},
$$

where $\bar{\rho}\left(T_{p} M\right)$ denotes the scalar curvature of the $n$-plane section $T_{p} M$ in the ambient manifold $\bar{M}$. Thus, we can say that $\rho(p)$ and $\bar{\rho}\left(T_{p} M\right)$ are the "intrinsic" and "extrinsic" scalar curvature of the submanifold at $p$, respectively.

We denote the set of unit vectors in $T_{p} M$ by $T_{p}^{1} M$, thus:

$$
T_{p}^{1} M=\left\{X \in T_{p} M: g(X, X)=1\right\} .
$$

Now, we recall the following result from [12]. 
Theorem 5.1. Let $M$ be an n-dimensional submanifold of a Riemannian manifold $\bar{M}$. Then the following statements are true:

(i). For $X \in T_{p}^{1} M$, we have:

$$
\operatorname{Ric}(X) \leq \frac{1}{4} n^{2}\|\mu\|^{2}+\overline{\operatorname{Ric}}_{\left(T_{p} M\right)}(X)
$$

where $\overline{\operatorname{Ric}}_{\left(T_{p} M\right)}(X)$ is the $n$-Ricci curvature of $T_{p} M$ at $X \in T_{p}^{1} M$ with respect to the ambient manifold $\bar{M}$.

(ii). The equality case of (5.5) is satisfied by $X \in T_{p}^{1} M$ if and only if:

$$
h(X, X)=\frac{n}{2} \mu(p) \quad \text { and } \quad h(X, Y)=0,
$$

for all $Y \in T_{p} M$ such that $g(X, Y)=0$.

(iii). The equality case of (5.5) holds for all $X \in T_{p}^{1} M$ if and only if either $p$ is a totally geodesic point or $n=2$ and $p$ is a totally umbilical point.

From Theorem 5.1, we immediately have the following:

Corollary 5.2. Let $M$ be an n-dimensional submanifold of a Riemannian manifold $\bar{M}$. For $X \in T_{p}^{1} M$ any two of the following three statements imply the remaining one:

(i) The mean curvature vector $\mu(p)$ vanishes.

(ii) The unit vector $X$ belongs to the relative null space $\mathcal{N}_{p}$.

(iii) The unit vector $X$ satisfies the equality case of (5.5), namely:

$$
\operatorname{Ric}(X)=\frac{1}{4} n^{2}\|\mu\|^{2}+\overline{\operatorname{Ric}}_{\left(T_{p} M\right)}(X) \text {. }
$$

From now on, let $\bar{M}$ be a generalized f.p.k.-space form. A submanifold $M$ of $\bar{M}$ is an integral submanifold if $\eta_{\alpha}(X)=0, \alpha=1, \ldots, s$, for every tangent vector $X$. A submanifold $M$ of $\bar{M}$ is an anti-invariant submanifold if $\varphi(T M) \subseteq T^{\perp} M$. An integral submanifold is identical with an anti-invariant submanifold normal to the structure vector fields $\xi_{1}, \ldots, \xi_{s}$. In particular case of $s=1$, an integral submanifold $M$ of a Sasakian manifold is a $C$-totally real submanifold [23]. It is known that [5] an $n$-dimensional integral submanifold $M$, of an $S$-manifold $\bar{M}$ of dimension $(2 n+s)$, is of constant curvature $s$ if and only if the normal connection is flat.

First, we give the following Lemma.

Lemma 5.3. Let $M$ be an n-dimensional integral submanifold of an f.p.k.-space form $\bar{M}^{2 m+s}(c)$. Let $\left\{e_{1}, \ldots, e_{n}\right\}$ be an orthonormal basis of the tangent space $T_{p} M$. Then:

$$
\begin{gathered}
\bar{K}_{i j}=F_{1}, \\
\overline{\operatorname{Ric}}_{T_{p} M}\left(e_{i}\right)=(n-1) F_{1}, \\
\left.\bar{\rho}_{(} T_{p} M\right)=\frac{1}{2} n(n-1) F_{1} .
\end{gathered}
$$

Proof. Equation (5.8) follows from (2.8). Using: $\overline{\operatorname{Ric}_{\left(T_{p} M\right)}}\left(e_{i}\right)=\sum_{j \neq i}^{n} \bar{K}_{i j}$ in (5.8), we

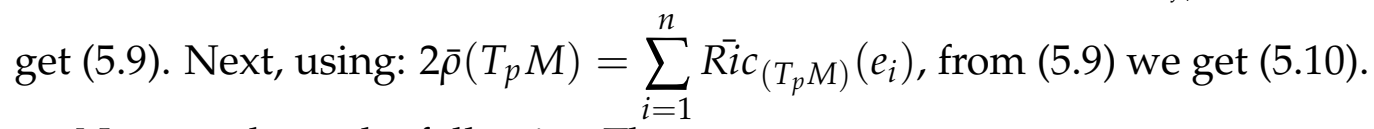

Now, we have the following Theorem. 
Theorem 5.4. If $M$ is an n-dimensional integral submanifold of an f.p.k.-space form $\bar{M}^{2 m+s}(c)$, then the following statements are true:

(i). For $X \in T_{p}^{1} M$, it follows that:

$$
\operatorname{Ric}(X) \leq \frac{1}{4}\left\{n^{2}\|\mu\|^{2}+4(n-1) F_{1}\right\} .
$$

(ii). The equality case of (5.11) is satisfied by $X \in T_{p}^{1} M$ if and only if (5.6) is true. If $\mu(p)=0, X \in T_{p}^{1} M$ satisfies equality in (5.11) if and only if $X \in \mathcal{N}_{p}$.

(iii). The equality case of (5.4) holds for all $X \in T_{p}^{1} M$ if and only if either $p$ is a totally geodesic point or $n=2$ and $p$ is a totally umbilical point.

Proof. Using (5.9) in (5.5), we find the inequality (5.11). Rest of the proof is straightforward.

By polarization, from Theorem 5.4, we drive:

Theorem 5.5. Let $M$ be an n-dimensional integral submanifold of an f.p.k.-space form $\bar{M}^{2 m+s}(c)$. Then the Ricci tensor Ric satisfies:

$$
\text { Ric } \leq \frac{1}{4}\left\{n^{2}\|\mu\|^{2}+4(n-1) F_{1}\right\} g .
$$

where $g$ is the induced Riemannian metric on $M$. The equality case of (5.12) is true if and only if either $M$ is totally geodesic submanifold or $M$ is a totally umbilical surface.

When $s=0$, we have the following two results:

Theorem 5.6. If $M$ is an $n$-dimensional totally real submanifold of an $2 m$-dimensional generalized complex space form $\bar{M}^{2 m}\left(f_{1}, f_{2}\right)$, then the following statements are true:

(i). For $X \in T_{p}^{1} M$, it follows that:

$$
\operatorname{Ric}(X) \leq \frac{1}{4}\left\{n^{2}\|\mu\|^{2}+4(n-1) f_{1}\right\} .
$$

(ii). The equality case of (5.13) is satisfied by $X \in T_{p}^{1} M$ if and only if (5.6) is true. If $\mu(p)=0, X \in T_{p}^{1} M$ satisfies equality in (5.13) if and only if $X \in \mathcal{N}_{p}$.

(iii). The equality case of (5.13) holds for all $X \in T_{p}^{1} M$ if and only if either $p$ is a totally geodesic point or $n=2$ and $p$ is a totally umbilical point.

It is known that (Theorem $4,[21]$ ) if $M$ is an $n$-dimensional compact minimal C-totally real submanifold of a Sasakian space form $\bar{M}^{2 m+1}(c) ; c>-3$, such that $M$ has positive sectional curvature, then $M$ is totally geodesic. Therefore, we have the following:

Theorem 5.7. An n-dimensional compact minimal C-totally real submanifold of a Sasakian space form $\bar{M}^{2 m+1}(c) ; c>-3$ with positive sectional curvature is Einstein manifold and satisfies:

$$
4 \text { Ric }=(n-1)(c+3) g
$$




\section{Minimality of integral submanifolds of maximum dimension}

We already know the following result ([5]). If $M$ is an $n$-dimensional minimal integral submanifold of any $(2 m+s)$-dimensional $S$-space form $\bar{M}(c)$, then the following four statements are equivalent:

(i). $M$ is totally geodesic.

(ii). $M$ is of constant curvature $\frac{1}{4}(c+3 s)$.

(iii). The Ricci tensor is $\frac{1}{4}(n-1)(c+3 s) g$.

(iv). The scalar curvature is $\frac{1}{4} n(n-1)(c+3 s)$.

Lemma 6.1. Let $M^{m}$ be an integral submanifold of a generalized f.p.k.-space form $\bar{M}^{2 n+s}(c)$. Then, we have:

(i). $g\left(\varphi\left(\bar{\nabla}_{X} \varphi\right) Y, Z\right)+g\left(\left(\bar{\nabla}_{X} \varphi\right) \varphi Y, Z\right)=0$, for any $X \in T \bar{M}$ and $Y, Z \in T M$.

(ii). $\bar{\nabla}_{X} \tilde{\xi}_{i} \in T^{\perp} M$, for any $X \in T \bar{M}$.

(iii). $A_{\xi_{\alpha}}=0$ for any $\alpha=1, \ldots, s$.

(iv). $A_{\varphi X} Y=A_{\varphi Y} X$, for any $X, Y \in T M$.

Proof. Covariantly differentiating $\varphi^{2}=-I+\sum_{i=1}^{s} \eta^{i} \xi_{i}$ with respect to any $X \in T \bar{M}$, we get:

$$
\varphi o\left(\bar{\nabla}_{X} \varphi\right)+\left(\bar{\nabla}_{X} \varphi\right) o \varphi=\sum_{i=1}^{s}\left(\bar{\nabla}_{X} \eta^{i}\right) \xi_{i}+\sum_{i=1}^{s} \eta^{i} \bar{\nabla}_{X} \xi_{i},
$$

which implies for an integral submanifold $M$ :

$$
g\left(\varphi\left(\bar{\nabla}_{X} \varphi\right) Y, Z\right)+g\left(\left(\bar{\nabla}_{X} \varphi\right) \varphi Y, Z\right)=0,
$$

for any $X \in T \bar{M}$ and $Y, Z \in T M$. This proves (i). Using $Y=\xi_{i}$ in above mentioned equation we get for any $Z \in T M$ :

$$
g\left(\varphi^{2}\left(\bar{\nabla}_{X} \tilde{\zeta}_{i}\right), Z\right)=0
$$

which implies:

$$
g\left(\bar{\nabla}_{X} \xi_{i}, Z\right)=\sum_{\alpha=1}^{s} \eta^{\alpha}\left(\bar{\nabla}_{X} \xi_{i}\right) g\left(\xi_{\alpha}, Z\right),
$$

for any $X \in T \bar{M}$ and $Z \in T M$. Since, $M$ is integral submanifold, we get:

$$
g\left(\bar{\nabla}_{X} \xi_{i}, Z\right)=0,
$$

for any $X \in T \bar{M}$ and $Z \in T M$. Namely, $\bar{\nabla}_{X} \xi_{i} \in T^{\perp} M$, which proves (ii). Now, we have:

$$
\bar{\nabla}_{X} \xi_{i}=-A_{\xi_{i}} X+\nabla_{X}^{\perp} \xi_{i}
$$

for any $X \in T \bar{M}$. That is, $A_{\xi_{i}}=0$, which proves our assertion in (iii). Finally, we have:

$$
g\left(A_{\varphi X} Y, X\right)=g(h(X, Y), \varphi X)=-g(X, \varphi h(X, Y))
$$

and:

$$
g\left(A_{\varphi Y} X, Y\right)=g(h(X, Y), \varphi Y)=-g(Y, \varphi h(X, Y))
$$

for any $X, Y \in T M$. Then, we get:

$$
A_{\varphi X} Y=-\varphi h(X, Y)=A_{\varphi Y} X
$$

which completes our proof for (iv).

Following the same arguments as in [5], we get: 
Theorem 6.2. If $M$ is an n-dimensional minimal integral submanifold of $(2 m+s)$ dimensional f.p.k.-space form $\bar{M}^{2 m+s}(c)$, then the following four statements are equivalent:

(i). $M$ is totally geodesic.

(ii). $M$ is of constant curvature $F_{1}$.

(iii). The Riccitensor is $(n-1) F_{1} g$.

(iv). The scalar curvature is $n(n-1) F_{1}$.

Theorem 6.3. Let $M$ be an $n$-dimensional integral submanifold of $(2 n+s)$-dimensional f.p.k.-space form $\bar{M}^{2 n+s}(c)$. If a unit vector of $T_{p}(M)$ satisfies the equality case of (5.11), then $\mu(p)=0$.

Proof. (see [15]) Choosing an orthonormal basis $\left\{e_{1}, \ldots, e_{n}\right\}$ of $T_{p} M$ such that $e_{1}$ satisfies the equality case of (5.11), we find $\left\{e_{n+1}=\varphi e_{1}, \ldots, e_{2 n}=\varphi e_{n}, e_{2 n+1}=\right.$ $\left.\xi_{1}, \ldots, e_{2 n+s}=\xi_{s}\right\}$ as an orthonormal basis of $T_{p}^{\perp} M$. From Lemma 6.1, $A_{\xi_{i}}=0$ for all $i \in\{1, \ldots, s\}$ and $A_{\varphi X} Y=A_{\varphi} X$ for $X, Y \in T M$. Then, by straightforward calculation and using (5.6), we have: $g\left(h\left(e_{1}, e_{1}\right), Z\right)=0$ where $Z \in T_{p}^{\perp} M$ and $Z=\sum_{j=1}^{n} a_{j} e_{n+j}+\sum_{\alpha=1}^{s} a_{\alpha} \xi_{\alpha}$. Using (5.6) results $\mu(p)=0$.

The maximum Ricci curvature function ([8]) on a Riemannian manifold $M$, denoted $\overline{\operatorname{Ri}}(p)$, is defined as:

$$
\overline{\operatorname{Ric}}(p)=\max \left\{\operatorname{Ric}(X): X \in T_{p}^{1} M\right\} .
$$

Now, in view of Theorem (6.3), we immediately have the following:

Theorem 6.4. Let $M$ be an $n$-dimensional integral submanifold of $(2 n+s)$-dimensional f.p.k.-space form $\bar{M}^{2 n+s}(c)$. Then:

$$
\overline{\text { Ric }} \leq \frac{1}{4}\left\{n^{2}\|\mu\|^{2}+4(n-1) F_{1}\right\} \text {. }
$$

If $M$ satisfies the equality case of (6.1) identically, then $M$ is a minimal submanifold and:

$$
\overline{\text { Ric }}=(n-1) F_{1} \text {. }
$$

Theorem 6.5. Let $M^{n}$ be a Lagrangian submanifold of a $2 n$-dimensional generalized complex space form $\bar{M}^{2 n}(c)$. Then:

$$
\overline{\text { Ric }} \leq \frac{1}{4}\left\{n^{2}\|\mu\|^{2}+4(n-1) f_{1}\right\} \text {. }
$$

If $M$ satisfies the equality case of (6.3) identically, then $M$ is a minimal submanifold and:

$$
\overline{\text { Ric }}=(n-1) f_{1} \text {. }
$$

From Theorem 6.4 we can state similar results for the cases of complex space form, generalized Sasakian space form, $S$-space form, Sasakian space form, Kenmotsu space form, cosymplectic space form and almost $C(\alpha)$-manifold, easily.

Following the arguments as in [8], we can prove:

Theorem 6.6. Let $M$ be an $n$-dimensional minimal integral submanifold of a $(2 n+s)$ dimensional f.p.k.-space form $\bar{M}^{2 n+s}(c)$. Then the following statements are true:

(i). The submanifold $M$ satisfies the equality case of (6.1) if and only if $\operatorname{dim}\left(\mathcal{N}_{p}\right) \geq 1$. 
(ii). If $\operatorname{dim}\left(\mathcal{N}_{p}\right)$ is a positive constant $d$, then $\mathcal{N}_{p}$ is completely integral distribution and $M$ is d-ruled, that is, for each $p \in M, M$ contains a d-dimensional totally geodesic submanifold $M^{\prime}$ of $\bar{M}(c)$ passing through $p$.

(iii). If the submanifold $M$ is also ruled, then it satisfies the equality case of (6.1) identically if and only if, for each ruling $M^{\prime}$ in $M$, the normal bundle $T^{\perp} M$ restricted to $M^{\prime}$ is a parallel normal subbundle of the normal bundle $T^{\perp} M^{\prime}$ along $M^{\prime}$.

Acknowledgment. The author is thankful to the referee for the helpful suggestions.

\section{References}

[1] P. Alegre and A. Carriazo, Structures on generalized Sasakian-space-forms, Differential Geom. Appl. 26, 656-666 (2008).

[2] D. E. Blair, Contact manifolds in Riemannian geometry, Lecture Notes in Math. 509, Springer-Verlag, 1976.

[3] D. E. Blair, Geometry of manifolds with structural group $U(n) \times O(s)$, J. Differ. Geom. 4, 155-167 (1970).

[4] D. E. Blair, Riemannian geometry of Contact and Symplectic Manifolds, Birkhauser, Boston, 2002.

[5] J. L. Cabrerizo, L. M. Fernández and M. Fernandez, On certain anti-invariant submanifolds of an S-manifold, Portugal. Math. 50, No. 1, 103-113 (1993).

[6] B. Y. Chen, Geometry of submanifolds, Marcel Dekker, New York, 1973.

[7] B. Y. Chen, Mean curvature and shape operator for isometric immersions in real space form, Glasg. Math. J., 38, 87-97 (1996).

[8] B. Y. Chen, On Ricci curvature of isotropic and Lagrangian submanifolds in complex space forms, Arch. Math. (Basel) 74, No. 2, 154-160 (2000).

[9] B. Y. Chen, Relations between Ricci curvature and shape operator for submanifolds with arbitrary codimensions, Glasg. Math. J., 41, No. 1, 33-41 (1999).

[10] M. Falcitelli and A. M. Pastore, Generalized globally framed f-space-forms, Bull. Math. Soc. Sci. Math. Roumanie, Tome 52(100) No. 3, 291-305 (2009).

[11] L. M. Fernández and M. B. Hans-Uber, New relationships involving the mean curvature of slant submanifolds in S-space-forms, J. Korean Math. Soc. 44, No. 3, 647-659 (2007).

[12] S. Hong and M. M. Tripathi, On Ricci curvature of submanifolds, Int. J. Pure Appl. Math. Sci. 2, No. 2, 227-245 (2005).

[13] D. Janssens and L. Vanhecke, Almost contact structure and curvature tensors, Kodai Math. J. 4, 1-27 (1981). 
[14] K. Kenmotsu, A class of almost contact Riemannian manifolds, Tohoku Math. J. II Ser. 24, 93-103 (1972).

[15] J.-S. Kim, M. K. Dwivedi and M. M. Tripathi, Ricci curvature of integral submanifolds of an S-space form, Bull. Korean Math. Soc. 44, No. 3, 395-406 (2007).

[16] J.-S. Kim, M. K. Dwivedi and M. M. Tripathi, Ricci curvature of submanifolds of an S-space form, Bull. Korean Math. Soc. 46, No. 5, 979-998 (2009).

[17] K. Matsumoto, I. Mihai, Ricci tensor of C-totally real submanifolds in Sasakian space forms, Nihonkai Math. J. 13, No. 2, 191-198 (2002).

[18] I. Mihai, Ricci curvature of submanifolds in Sasakian space forms, J. Austral. Math. Soc. 72, No. 2, 247-256 (2002).

[19] J. A. Oubiña, New classes of almost contact metric structure, Publ. Math. Debercen 32, 187-193 (1985).

[20] F. Tricerri and L. Vanhecke, Curvature tensors on almost Hermitian manifolds, Trans. Amer. Math. Soc. 267, 365-398 (1981).

[21] D. Van Lindt, P. Verheyen and L. Verstraelen, Minimal submanifolds in Sasakian space forms, J. Geom. 27, No. 2, 180-187 (1986).

[22] L. Ximin, On Ricci curvature of C-totally real submanifolds in Sasakian space forms, Proc. Indian Acad. Sci. (Math. Sci.), V. 111, No. 4, 399-405 (2001).

[23] S. Yamaguchi, M. Kon and T. Ikawa, C-totally real submanifolds, J. Differential Geometry, 11, No. 1, 59-64 (1976).

[24] K. Yano, On a structure defined by a tensor field $f$ satisfying $f^{3}+f=0$, Tensor, New Ser. 14, 99-109 (1963).

[25] K. Yano and M. Kon, Structures on manifolds, World Scientific Press (1984).

Department of Mathematics, Jamia Millia Islamia, New Delhi-110025, India

E-mail address: mahmoodjaafari280@gmail.com 